\title{
Phase Diagram of Dipolar Hard and Soft Spheres: Manipulation of Colloidal Crystal Structures by an External Field
}

\author{
Antti-Pekka Hynninen and Marjolein Dijkstra \\ Soft Condensed Matter, Debye Institute, Utrecht University, Princetonplein 5, 3584 CC Utrecht, The Netherlands
}

(Received 13 August 2004; published 8 April 2005)

\begin{abstract}
Phase diagrams of hard and soft spheres with a fixed dipole moment are determined by calculating the Helmholtz free energy using simulations. The pair potential is given by a dipole-dipole interaction plus a hard-core and a repulsive Yukawa potential for soft spheres. Our system models colloids in an external electric or magnetic field, with hard spheres corresponding to uncharged and soft spheres to charged colloids. The phase diagram of dipolar hard spheres shows fluid, face-centered-cubic (fcc), hexagonalclose-packed (hcp), and body-centered-tetragonal (bct) phases. The phase diagram of dipolar soft spheres exhibits, in addition to the above mentioned phases, a body-centered-orthorhombic (bco) phase, and it agrees well with the experimental phase diagram [Nature (London) 421, 513 (2003)]. Our results show that bulk hcp, bct, and bco crystals can be realized experimentally by applying an external field.
\end{abstract}

DOI: 10.1103/PhysRevLett.94.138303

Dispersions of colloidal particles with a dielectric constant mismatch with the surrounding solvent, that are subject to an external electric field, acquire a dipole moment parallel to the field. Similarly, para- or diamagnetic colloids in a solvent, subject to an external magnetic field acquire a dipole moment parallel to the field [1]. These suspensions are called electro- (ER) and magnetorheological (MR) fluids, because their rheological properties can be controlled by the external field $[2,3]$. If the electric field exceeds a critical value, the fluid turns into a solid whose yield stress increases upon increasing the field. ER and MR fluids have potential use in industrial applications as hydraulic valves, clutches, brakes, etc., Moreover, the possibility to tune the crystal structure of these suspensions by the applied electric field makes these suspensions appealing for photonic applications [4-6].

The phase behavior of these systems is determined to first order by a dipole-dipole interaction. As the dipoledipole interaction favors orientations where the dipoles are head to toe, particles tend to form strings in the direction of the field. The equilibrium structure of ER fluids has been the subject of many experimental [4,5,7-9], theoretical [10-14], and simulation studies [15-17], and by now, it has been widely accepted, that the high-field ground state is a body-centered-tetragonal (bct) crystal [see Figs. 1(a) and $1(\mathrm{~b})]$. The bct crystal is also the high-field ground state of MR fluids [3,18].

Despite the great number of theoretical and simulation studies on dipolar hard-spheres [10-17], the full phase behavior, including effects due to the entropy, has so far not been presented. In fact, many of the previous studies focused on the high-field limit, where the energy term of the free energy dominates the phase behavior and the entropy part plays no role. In this Letter, we present, for the first time, a full phase diagram, that spans from zero field, where the system is entropy dominated, up to the high-field limit, where the system is energy dominated. In the limit of zero field, the well-known hard-sphere phase
PACS numbers: 82.70.Dd, 64.70.-p, 83.80.Gv

behavior is recovered, while in the limit of high field, the system shows stable bct and broad fluid-bct coexistence. More surprisingly, we find that, between the two extremes, the high-density stable state is a hexagonal-close-packed (hcp) phase [see Fig. 1(c)], which is known to be unstable in bulk [19].

In the case of dipolar soft spheres, a phase diagram analogous to the dipolar hard-sphere case is obtained, that shows, in addition, a body-centered-orthorhombic (bco) phase [see Figs. 1(a) and 1(b)]. The dipolar soft-

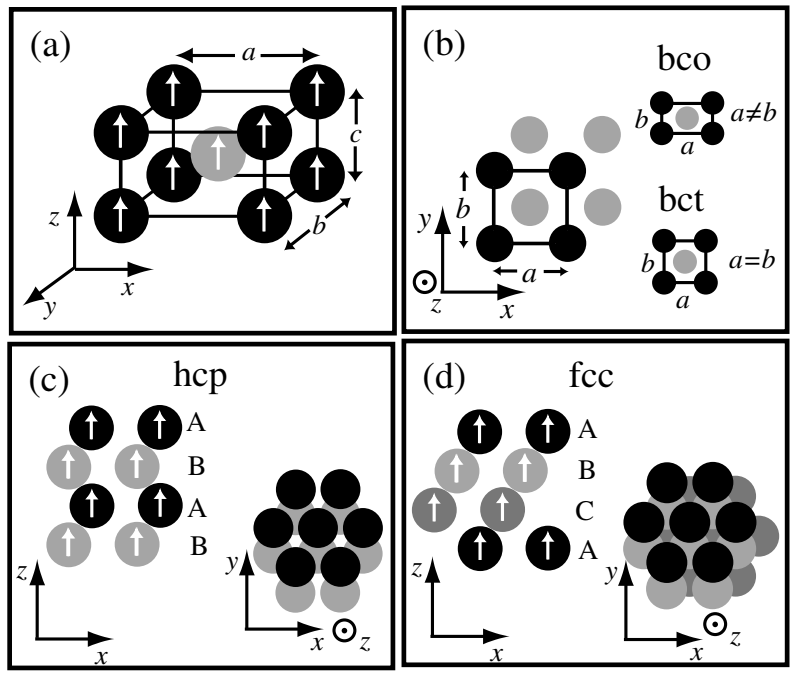

FIG. 1. (a) Body-centered structure in three dimensions, whose conventional unit cell is $a \times b \times c$. The field is along the $z$ axis. The white arrows show the direction of the fieldinduced dipole moments. The bct structure corresponds to $a=$ $b \neq c$, and the bco to $a \neq b, c \neq a$, and $c \neq b$. (b) Top view of the body-centered structure, that can be constructed by placing strings of particles shifted by $c / 2$ into two interpenetrating rectangular lattices. (c),(d) The hep and fcc structures shown in side and top views. The hcp structure has $A B$ stacking of the hexagonal planes, and the fcc $A B C$. 
sphere system can be used to model charged colloids in a high-frequency electric field; a model system studied recently by Yethiraj and van Blaaderen [4,5,9]. We find good agreement with the experimental phase diagram in Ref. [4] and explain the stability of the bco phase [see Figs. 1(a) and 1 (b)] based on simple energy arguments.

In our model, we assume an electric or magnetic field parallel to the $z$ axis, that gives rise to a dipole-dipole pair interaction given by

$$
\beta u_{\text {dip }}\left(\mathbf{r}_{i j}\right)=\frac{\gamma}{2}\left(\frac{\sigma}{r_{i j}}\right)^{3}\left(1-3 \cos ^{2} \theta_{i j}\right),
$$

where $\sigma$ is the diameter of the particles, $\beta=1 / k_{B} T, \mathbf{r}_{i j}$ is the vector separating particles $i$ and $j$, and $\theta_{i j}$ is the angle that $\mathbf{r}_{i j}$ forms with the $z$ axis. In the case of electric dipoles, the dimensionless prefactor $\gamma$ in Eq. (1) is given by $\gamma=$ $\mathbf{p}^{2} / 2 \pi \epsilon_{s} \sigma^{3} k_{B} T$ in mksa (units of measure), where $\alpha=$ $\left(\epsilon-\epsilon_{s}\right) /\left(\epsilon+2 \epsilon_{s}\right), \epsilon$, and $\epsilon_{s}$ are the dielectric constants of the particles and the solvent, respectively, and $\mathbf{p}=$ $\frac{\pi}{2} \alpha \epsilon_{s} \sigma^{3} \mathbf{E}_{\text {loc }}$ is the dipole moment induced by the local electric field $\mathbf{E}_{\text {loc }}=\mathbf{E}+\mathbf{E}_{\text {dip }}$. Here $\mathbf{E}$ is the external field and $\mathbf{E}_{\mathrm{dip}}$ is the field induced by the other dipoles $[10,16,17]$. In the case of magnetic dipoles, we have $\gamma=$ $\mathbf{m}^{2} / 2 \pi \sigma^{3} k_{B} T$, where $\mathbf{m}=\frac{\pi}{2} \sigma^{3} \mathbf{H}_{\mathrm{loc}}\left(\mu-\mu_{s}\right) /\left(\mu+2 \mu_{s}\right)$ is the induced magnetic moment, $\mu$ and $\mu_{s}$ are the magnetic permeabilities of the particles and the solvent, and $\mathbf{H}_{\text {loc }}=\mathbf{H}+\mathbf{H}_{\text {dip }}$ is the local magnetic field [3].

In the case of dipolar soft spheres, we use a repulsive hard-core Yukawa potential given by

$$
\beta u_{\mathrm{Y}}(r)=\left\{\begin{array}{cc}
\frac{\epsilon \exp [-\kappa(r-\sigma)]}{r / \sigma}, & r \geq \sigma \\
\infty, & r<\sigma
\end{array}\right.
$$

where $\epsilon=Z^{2} /(1+\kappa \sigma / 2)^{2}\left(\lambda_{B} / \sigma\right)$ is a constant prefactor depending on the colloidal charge number $Z$, Debye screening length $\kappa^{-1}$, and Bjerrum length $\lambda_{B}=$ $e^{2} / \epsilon_{s} k_{B} T$. Equation (2) is the pair potential given by the Derjaguin-Landau-Verwey-Overbeek theory for charged colloids [20]. Since we are interested in systems where the van der Waals attraction is very small due to refractive index matching, we have neglected it in Eq. (2). Phase diagram of Yukawa particles has stable fluid, body-centered-cubic (bcc), and face-centered-cubic (fcc) phases [21].

We perform Monte Carlo (MC) simulations in the canonical ensemble $(N V T)$, where we fix the number of particles $N$, the volume $V$, and the temperature $T$ [22]. The simulation box is periodic in all three directions. Typical number of particles in our simulations is $N=$ 144-300, and cubic (or nearly cubic) simulation boxes are used. Finite size effects were checked to be insignificant by doubling the number of particles for a couple of state points. The Ewald summation method is used to calculate $u_{\mathrm{dip}}\left(\mathbf{r}_{i j}\right)$ [22], and the Gaussian width is optimized according to the analytic estimates given in Ref. [23]. Both the Yukawa and the real-space part of the dipolar potential are truncated at half of the shortest box side length. The phase behavior is determined by Helmholtz free energy calculations using thermodynamic integration: we use the so-called $\lambda$ integration for the fluid phase and the Frenkel-Ladd method for the solid phase [22]. Phase coexistence regions are determined using the common tangent construction.

Figure 2 shows the phase diagram of the dipolar hard spheres in the dipole moment strength $\gamma$-packing fraction $\eta=(\pi / 6) \sigma^{3} N / V$ representation. At $\gamma=0$, the wellknown hard-sphere fluid-fcc coexistence is recovered, which at $\gamma \gtrsim 1$ turns into fluid-hcp coexistence. Figure 2 shows that the hcp phase dominates the high-density part of the phase diagram for $\gamma \geq 2$. The hcp and fcc phases are not entirely symmetric, but are slightly compressed in the $z$ direction (less than 10\%), because of the dipole-dipole interactions. At $\gamma \approx 8.0$, the system phase separates into a (string) fluid phase and bct phase. The inset (a) in Fig. 2 shows a snapshot of the string fluid phase, where the particles are aligned in strings parallel the $z$ axis. The fluid-bct phase coexistence region broadens with increasing dipole moment strength and at $\gamma=13.1$, the coexisting fluid phase is a very dilute gas phase (or a void, i.e. $\eta \approx 0$ ). The inset (b) in Fig. 2 shows a snapshot taken inside the gas-bct coexistence region.

At $\gamma \approx 38$, the bct phase has a packing fraction close to the maximum body-centered packing $\eta_{\mathrm{bc}}^{\max } \approx 0.698$. Snapshot (c) in Fig. 2 illustrates the gas-bct phase separation at dipole moment strength $\gamma=26.1$. We expect at $\gamma>38$, coexistence between a void and a bct phase at $\eta_{\mathrm{bc}}^{\max }$, and a stable hcp phase for $\eta>\eta_{\mathrm{bc}}^{\max }$.

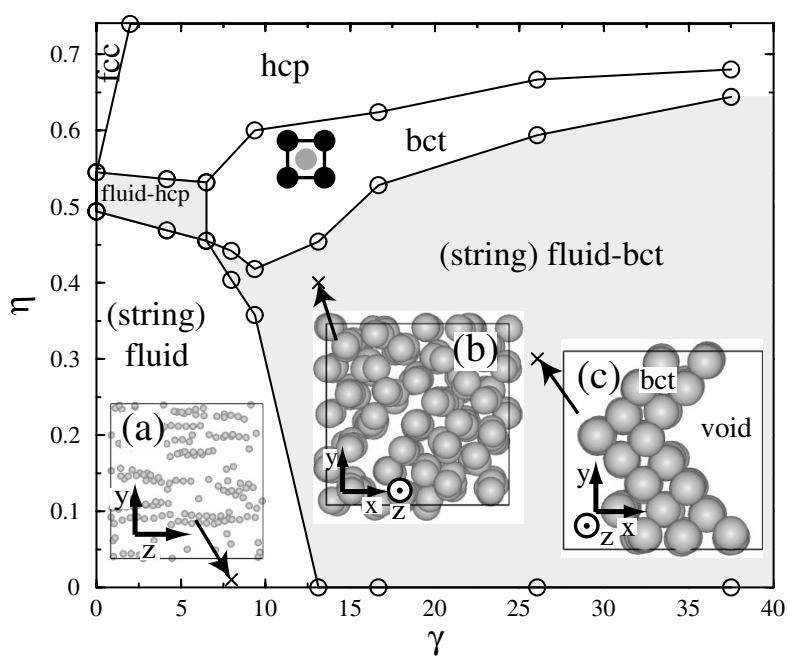

FIG. 2. Phase diagram of dipolar hard spheres in the dipole moment strength $\gamma$-packing fraction $\eta$ representation. The circles denote points where the phase boundary was determined and the gray areas denote coexistence regions (where the tie lines are vertical). Snapshots are taken (a) in the string fluid phase ( $\gamma=8.0, \eta=0.01)$, and (b),(c) in the fluid-bct coexistence region at $(\gamma=13.1, \eta=0.4)$ and at $(\gamma=26.1, \eta=0.3)$, respectively. 
In the case of dipolar soft spheres, we use parameters $\kappa \sigma=10.0$ and $\epsilon=12.54$, that for a solvent with Bjerrum length $\lambda_{B} / \sigma=0.005$ corresponds to a bare charge of $Z=$ 300 . The phase diagram of the dipolar soft spheres is shown in Fig. 3. At $\gamma=0$, Fig. 3 shows a fluid-fcc coexistence with coexisting packing fractions $\eta_{\text {fluid }}=0.31$ and $\eta_{\mathrm{fcc}}=0.32$. For $\gamma \gtrsim 17$, the hcp phase dominates the high-density part of the phase diagram. As can be seen from Fig. 3, increasing the dipole moment strength reduces the stability region of the hcp phase and, at $\gamma \geq 100$, the hcp phase is only stable at $\eta>\eta_{\mathrm{bc}}^{\max }$. In the range $4<\gamma<$ 67 , we find a new colloidal crystal structure, i.e., the asymmetric bco phase, which is a result of the combination of the soft repulsive interactions and the induced dipolar interactions. At $\gamma \gtrsim 50$, a stable bct phase is found at high $\eta$, while fluid-bct coexistence is found at low $\eta$. We expect that the fluid-bct coexistence broadens further when $\gamma>$ 180 , until the bct phase reaches the maximum packing $\eta_{\mathrm{bc}}^{\max }$.

In Fig. 3, the upper horizontal axis gives the (root mean square) electric field strength. The $\gamma$ to $E_{\mathrm{rms}}$ conversion is done using [24]

$$
E_{\mathrm{rms}}=2 \frac{\left|1-\alpha \frac{\pi}{6}\right|}{|\alpha|} \sqrt{\frac{k_{B} T \gamma}{\epsilon_{s} \sigma^{3} \pi}}
$$

where we used parameter values that correspond to the experimental system of Ref. [4]: $\alpha=-0.105, T=300 \mathrm{~K}$, $\epsilon_{s}=5.6$, and $\sigma=2 \mu \mathrm{m}$. This allows us to compare directly the two phase diagrams, and we find a remarkably

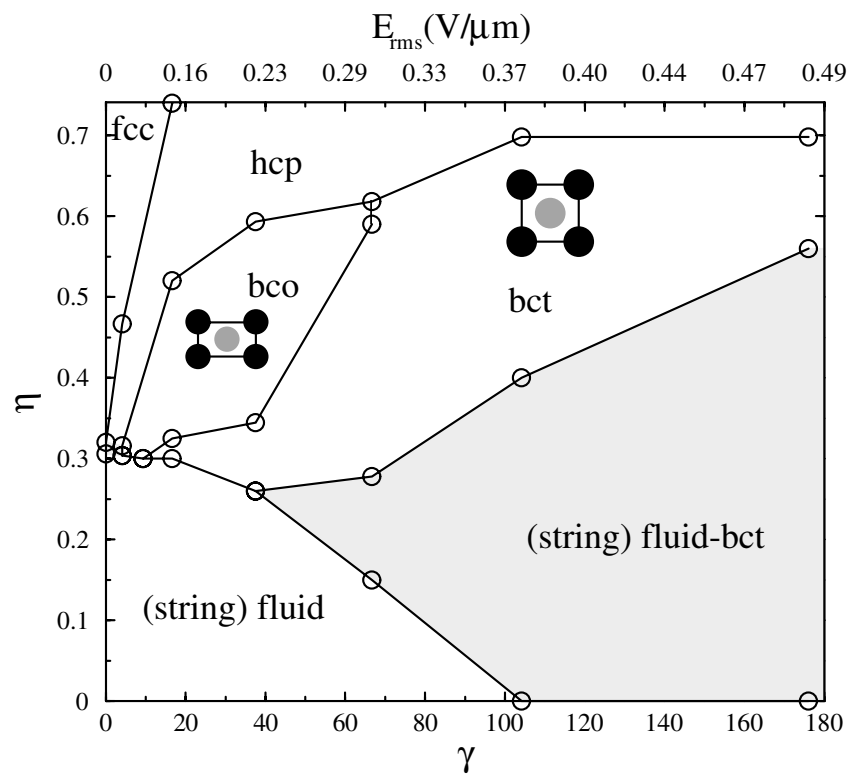

FIG. 3. Phase diagram of dipolar soft spheres with parameters $\kappa \sigma=10.0$ and $\epsilon=12.54$ in the (dimensionless dipole moment strength $\gamma$, packing fraction $\eta$ ) representation. The circles denote points where the phase boundary was determined and the gray area denotes the coexistence region (where the tie lines are vertical). good structural agreement. Both phase diagrams show, at low field strength, the same sequence of fluid, bct [25] and bco phases upon increasing $\eta$, and at high fields, coexistence between a gas (void) and a bct phase [26].

The bct and bco phases are explained as follows. Because of dipolar interactions, the particles form strings in the $z$ direction. If two strings are close to each other, it is favorable to shift one string by $c / 2$ in the $z$ direction with respect to the other string, as the dipole-dipole interaction favors configurations with small angles $\theta$ in Eq. (1). Consequently, two kind of strings, say $A$ and $B$, are obtained. In Fig. 1(b), $A$ strings are black and $B$ strings are gray. The interactions between the strings are such that similar strings $(A-A$ and $B-B)$ repel each other more than dissimilar strings $(A-B)$. This is why the bet is stable: it minimizes the $A-B$ distance. Soft Yukawa repulsion of $A-A$ strings and $A-B$ strings are very similar ( $A-B$ being slightly weaker), and it favors configurations where all neighboring strings have almost equal distances. This is achieved by increasing the ratio $a / b$ : In a hexagonal state (which is the ground state if all strings are similar) all nearest neighbors distances are equal and $a / b=\sqrt{3} \approx 1.73$ (see the illustrations in Fig. 4). Hence, the bco phase.

Figure 4 shows the change in Madelung energy (energy per particle of an ideal lattice) $\Delta U_{\mathrm{M}}(a / b)=U_{\mathrm{M}}(a / b)-$ $U_{\mathrm{M}}(1)$ of a bco crystal (with $c=\sigma$ ) at packing fractions $\eta=0.27,0.4$, and 0.5 . At $\eta=0.27$, the minimum of the Madelung energy is at $a / b=1$ and, therefore, the ground state is bct. At $\eta=0.4$ and 0.5 , the minimum is at $a / b \approx$ 1.4, meaning, that the ground state is bco. Splitting $\Delta U_{\mathrm{M}}(a / b)$ into a Yukawa and a dipolar part as

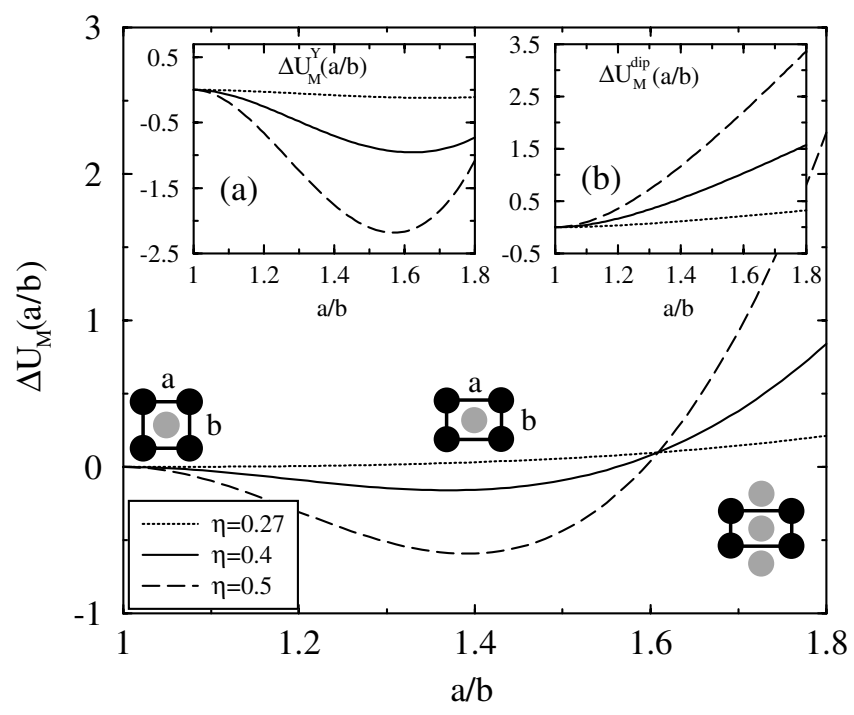

FIG. 4. Change in the Madelung energy $\Delta U_{\mathrm{M}}(a / b)=$ $U_{\mathrm{M}}(a / b)-U_{\mathrm{M}}(1)$ of a bco crystal when $a / b$ is increased from one. The results are for dipolar soft spheres with $\kappa \sigma=$ $10.0, \epsilon=12.54$, and $\gamma=37.5$, at packing fractions $\eta=0.27$, 0.4 , and 0.5 . The insets (a) and (b) show the soft and dipolar parts of the Madelung energy change, respectively. 
$\Delta U_{\mathrm{M}}(a / b)=\Delta U_{\mathrm{M}}^{\mathrm{Y}}(a / b)+U_{\mathrm{M}}^{\mathrm{dip}}(a / b)$ [see Fig. 4(a) and 4(b)], we observe that $\Delta U_{\mathrm{M}}^{\mathrm{dip}}(a / b)$ increases monotonically with $a / b$, while $\Delta U_{\mathrm{M}}^{\mathrm{Y}}(a / b)$ has a minimum at $a / b>1.5$. Thus, the bco phase is stabilized by the soft repulsion.

The reason that the hcp phase is more stable than the fcc phase is due to the difference between the Madelung energies of the two structures: The hop structure with the orientation shown in Fig. 1(c) has a Madelung energy $U_{\mathrm{M}}(\mathrm{hcp})=-0.37066 \times 4 \gamma$ (as in Refs. [8,17]), while the fcc structure (whose energy is independent of the orientation) has a Madelung energy $U_{\mathrm{M}}(\mathrm{fcc})=$ $-0.37024 \times 4 \gamma$ (as in Refs. [10,17]). Although the Madelung energy difference between hcp and fcc is small, $1.7 \gamma \times 10^{-3} k_{B} T$ per particle, so is their zero-field free energy difference: For hard spheres [19], the free energy per particle of the fcc is about $1 \times 10^{-3} k_{B} T$ lower than that of the hcp. For dipolar hard spheres, the hcp is expected to be stable for $\gamma \gtrsim 1$, which is consistent with Fig. 2 .

In order to study the effect of higher multipole moments on the stability of the hcp phase, we calculate the exact effective dielectric constants of the hcp and fcc structures using the method described in Ref. [27]. Knowing the effective dielectric constant $\epsilon_{\text {eff }}$, the total Coulomb energy per particle can be calculated from $-\left(\pi \sigma^{3} \mathbf{E}^{2} / 12 \eta k_{B} T\right) \times$ $\left(\epsilon_{\text {eff }}-\epsilon_{s}\right)$. According to the exact calculation, in the region where $\epsilon / \epsilon_{s}<1$ [28], the higher multipole moments make the hcp structure even more favorable than in the dipole approximation, and in the region where $\epsilon / \epsilon_{s}>1$, the hcp is always more stable than the fcc, although the higher multipole moments reduce the energy difference between the two phases with respect to the dipole approximation. This gives us confidence that the stable hcp phase is not an artifact of the dipole approximation but robust, and might be observed experimentally. As the hop phase is difficult to realize in bulk colloidal suspensions [29], this finding is of great importance.

In conclusion, we studied the phase behavior of dipolar hard and soft spheres as a function of the dipole moment strength $\gamma$ and the packing fraction $\eta$. We found very rich phase diagrams with stable regions of (string-) fluid, fcc, hcp, and bct phases, and regions of fluid-hcp and fluid-bct coexistence, for dipolar hard spheres. For dipolar soft spheres, we also found a stable region of the asymmetric bco phase. We explain the stability of the bco phase based on simple energy considerations. We find, that the hcp phase is more stable than the fcc phase, because of its lower energy, and more stable than the bct phase, because of its higher entropy. Our results show that bulk hcp, bct, and bco crystals, can be stabilized and therefore realized experimentally by applying an external field. We stress that these crystal phases are unstable in the absence of a field.

We thank A. van Blaaderen, J.H.J. Thijssen, A. Yethiraj, and R. van Roij for fruitful discussions. This work is part of the research program of the "Stichting voor Fundamenteel Onderzoek der Materie (FOM)", which is financially supported by the "Nederlandse Organisatie voor Wetenschappelijk Onderzoek (NWO)". We thank the Dutch National Computer Facilities foundation for access to the SGI Origin3800 and the High Performance Computing group of Utrecht University for computer time.

[1] R. Bubeck et al., Phys. Rev. Lett. 82, 3364 (1999).

[2] M. Parthasarathy and D. Klingenberg, Mater. Sci. Eng., R 17, 57 (1996).

[3] R. Tao, J. Phys. Condens. Matter 13, R979 (2001).

[4] A. Yethiraj and A. van Blaaderen, Nature (London) 421, 513 (2003).

[5] A. Yethiraj et al., Adv. Mater. 16, 596 (2004).

[6] R. Tao and D. Xiao, Appl. Phys. Lett. 80, 4702 (2002).

[7] T. Chen et al., Phys. Rev. Lett. 68, 2555 (1992).

[8] U. Dassanayake et al., J. Chem. Phys. 112, 3851 (2000).

[9] A. Yethiraj et al., Phys. Rev. Lett. 92, 058301 (2004).

[10] R. Tao and J. M. Sun, Phys. Rev. Lett. 67, 398 (1991).

[11] W. R. Toor and T.C. Halsey, Phys. Rev. A 45, 8617 (1992).

[12] R. Friedberg and Y.-K. Yu, Phys. Rev. B 46, 6582 (1992).

[13] H. J.H. Clercx and G. Bossis, Phys. Rev. E 48, 2721 (1993).

[14] B. Groh and S. Dietrich, Phys. Rev. E 63, 021203 (2001).

[15] R. Tao and J. M. Sun, Phys. Rev. A 44, R6181 (1991).

[16] R. Tao, Phys. Rev. E 47, 423 (1993).

[17] J.E. Martin et al., J. Chem. Phys. 108, 3765 (1998).

[18] L. Zhou et al., Phys. Rev. Lett. 81, 1509 (1998).

[19] A. D. Bruce et al., Phys. Rev. Lett. 79, 3002 (1997).

[20] B. Derjaguin and L. Landau, Acta Physicochim URSS 14, 633 (1941); E. J.W. Verwey and J. Th. G. Overbeek, Theory of the Stability of Lyotropic Colloids (Elsevier, Amsterdam, 1948).

[21] M. O. Robbins et al., J. Chem. Phys. 88, 3286 (1988).

[22] D. Frenkel and B. Smit, Understanding Molecular Simulations (Academic, New York, 2002), 2nd ed.

[23] Z. Wang and C. Holm, J. Chem. Phys. 115, 6351 (2001).

[24] For the derivation of Eq. (3), see, e.g., Refs. [10,16,17]. We have assumed a cubic lattice, for which the local field is given by $\mathbf{E}_{\text {loc }}=\mathbf{E} /(1-\alpha \pi / 6)$ [10].

[25] In Ref. [4], the bct phase is called a space-filling tetragonal (sft) phase.

[26] In the phase diagram of Ref. [4], the "non-space-filling bct" phase, which consists of small bct crystallites with voids, corresponds with our gas-bct coexistence. Experimentally, one observes a microphase separation instead of a macroscopic phase separation, which depends strongly on the kinetics and dynamics of the phase separation and on how fast the electric field is switched on.

[27] H. Ma et al., Phys. Rev. Lett. 77, 2499 (1996); Adv. Phys. 52, 343 (2003).

[28] The experimental systems of Ref. [4] have $\epsilon / \epsilon_{s} \approx 0.7$.

[29] The hcp order can be achieved by colloidal epitaxy: J. P. Hoogenboom et al., Phys. Rev. Lett. 90, 138301 (2003). 